\title{
Aufzählung der von Herrn Professor F. Sahlberg in Brasilien gesammelten Histeriden \\ von
}

Joh. Schmidt.

Die im Folgenden besprochenen Histeriden wurden vor fast einem halben Jahrhundert (in den Jahren 1849 und 50) im District von Rio Janeiro gesammelt. Die grosse Zahl der Arten, von denen damals kaum der fünfte Theil bekannt war, sowie die vielen interessanten kleinen Species zeugen von dem Eifer und der Findigkeit ihres Entdeckers. Im Laufe der Zeit sind theils nach anderweit aufgefundenen Exemplaren, theils auch nach hier- und dorthin abgegebenen Stücken der Sahlberg'schen Sammlung die meisten Arten beschrieben worden, immerhin bleiben noch etwa ein Dutzend Nova, darunter ein sehr merkwürdiges, jedenfalls myrmecophiles Genus. Auch Marseul scheint eine Anzahl der von Sahlberg entdeckten Arten durch Bohemann erhalten und im Supplement seiner Monographie - 1860-62 - publicirt zu haben. Die meisten, als deren Fundort er Rio Janeiro angiebt, fanden sich noch in der mir vorliegenden Sammlung, einige wenige waren nicht mehr vorhanden. Um ein vollständiges Bild des einstigen Umfangs dieser schönen Collektion zu geben, habe ich auch diese, mit einem *) bezẹichnet, mit aufgeführt. Schliesslich kann ich nicht umhin, Herrn Professor J. Sahlberg für die freundliche Mittheilung der Sammlung, sowie für die Liebenswürdigkeit zu danken, mit welcher er mir auch die Unica überlassen hat.

1. Tryponaeus thoracicus F. Syst. El. II, p. 385. - Petropolis.

2. Tryponaeus volvulus Er. Jahrb. 1834, p. 200. - Boa Sorta.

3. Tryponaeus bimaculatus Fr. Jahrb. 1834, p. 201. Boa Sorta. 
4. Tryponaeus Petropolitanus n. sp. - Petropolis.

․ Cylindricus, nigro-brunneus, nitidus; fronte opaca, subimpressa, rostro brevi convexo obtuso; pronoto antice sat dense, postice minus punctato, stria marginali breviter intervupta; elytris minute, ad suturam densius punctatis; pygidio brevi conico acuminato, sat dense punctulato; prosterno antice paululum angustato mesosternoque marginatis vix perspicue, metasterno distinctius punctulatis. - Long. $4 \mathrm{~mm}$.

6. Exemplar unicum capite pygidioque orbatum exstat. Thorace subtilius punctato, ante scutellum fere laevi, impressione pone caput lunata tuberculisque duobus minutis, paulo pone medium foveolato; sterno vix distincte punctulato, prosterno paululumlatiore.

Eine ausführliche Beschreibung dieser und der folgenden Art hoffe ich demnächst in einer Revision der Gattung Tryponaeus zu geben.

5. Tryponaeus obesus n. sp. - Petropolis.

오. Cylindricus, brevis, niger pedibus lrunneis. Fronte impressa, dense punctata, rostro obtuso; pronoto parum dense punctato, stria marginali vix interrupta; elytris minute sparsim punctulatis; pygidio dense punctulato, apice rotundato depresso brevissime piloso; prosterno parallelo lateribus acute elevatis; mesosterno duplo latiore stria antice integra, basi evanescente, minute, metasterno densius distinctius punctulato. - Long. $4^{1}{ }_{2} \mathrm{~mm}$.

6. Tryponaeus plagiatus Lew. Ann. a. Mag. N. H. 1891, p. 401. - Petropolis.

7. Phylloma corticale F. Syst. El. I, p. 91.--Boa Sorta, Petropolis.

8. Hololepta excisa Mars. Mon. 1853, p. 149, t. 4, f. 6. - Rio Janeiro.

9. Hololepta cubensis Er. Jahrb. 1834, p. 92. - Boa Sorta.

Ein recht kleines Exemplar, sonst aber mit Stücken von Cuba übereinstimmend.

10* Hololepta Januarii Mars. Mon. 1860, p. 602.

11. Hololepta humilis Payk. Mon. p. 109. - Boa Sorta.

12. Hololepta curta Mars. Mon. 1853, p. 187. - S. Rita.

13. Lioderma quadridentatum F. Ent. Syst. I, p. 74. S. Rita, Petropolis.

14. Lioderma punctulatum Mars. Mon. 1853, p. 208, t. 5, f. 6 $=$ Hololepta Sahlbergi Lew. Ann. a. Mag. N. H. 1885, p. 205. - Boa Sorta.

Da unter der von Lewis angegebenen Nr. ein Exemplar des i. punctulatum Mars. steckte, kann ich an der Identität der beiden nicht zweifeln. Die Beschreibungen stimmen vollständig. Lewis 
vergleicht die Art mit $L$. rimosum und minutum, stellt sie aber des breiten Prosternums wegen zu Hololepta, während Marseul sie der gezähnten Unterkante der Hinterschienen wegen mit Lioderma vereinigt; ein weiterer Beweis für die Unhaltbarkeit der letzteren Gattung.

15. Omalodes omega Kirby. Trans. Linn. Soc. XII, p. 394. S. Rita.

16. Omalodes angulatus F. Syst. El. I, p. 85. - Boa Sorta, Petropolis.

17. Omalodes foveola. Er. Jahrb. 1834, p. 120. - S. Rita.

18. Omalodes brasilianus Mars. Mon. 1853, p. 513. - Petropol. 19. Omalodes serenus Er. Jahrb. 1834, p. 123. - Boa Sorta, S. Rita.

\section{Hypobletus n. gen.}

Corpus ovatum plus minusve depressum; caput retractum, clypeus emarginatus, labrum valde transversum curvatum; antennae sub frontis margine insertae; fossa sub angulo thoracis; pronotum antice sinuatum, marginatum; scutellum distinctum, elytra striata; pygidium declive; prosternum lubatum, basi rotundatum, mesosternum late sinuatum vel subbisinuatum; tibiae anticae extus pluridentatae, fovea tarsali recta, extus haud marginata; tibiae posticae subdilatatae, extus vix armatae, spinulis 2-3 tantum versus apicem.

Ich stelle diese Gattung für eine Reihe bisher zu Apobletes und Platysoma gestellter südamerikanischer Arten auf. Es sind dies: A pobl. subridens Mrs., ridens Mrs., parensis Mrs., semirufus Lew, connectens Lew; Platysoma delicatum Lew., wozu noch die unten folgende und 2 demnächst $\mathrm{zu}$ beschreibende Bolivianische Species kommen. Mit Platysoma oder Apobletes können dieselben wegen der graden, aussen nicht scharf begrenzten Tarsalgruben nicht vereinigt werden. Diese beiden Gattungen scheinen überhaupt in südlichen Amerika zu fehlen, bis auf die cylindrische Platysoma-Form, welche in Mexico und Parà einige Repräsentanten zählt. Hypobletus steht dem Phelister viel näher und eine scharfe Unterscheidung von diesem vielgestaltigen Genus ist nicht leicht. Das Mesosternum ist ja anscheinend bei Hypobletus sehr verschieden, im Bogen von einer Ecke zur andern ausgerandet; bei einzelnen Arten ist aber diese Ausrandung schwach zweibuchtig und andrerseits kennen wir auch Phelister mit einfach ausgerandetem Mesosternum. Dagegen fehlt der hintere Querstreif des Mesosternum, den sämmtliche bekannten Phelister besitzen. Das Epistom ist ausgerandet und die Oberlippe schliesst sich in Form eines ganz schmalen Bogens an dasselbe an; 
die Hinterschienen haben über der Spitze höchstens 1 oder 2 feine Dörnchen; hierin ist die Gattung Baconia ähnlich, dieselbe besitzt aber ganz andre Vorderschienen und eine nicht gebogene Oberlippe. Die Streifen-Sculptur der Oberseite besitzt einige Eigenthümlichkeiten, die bei andern Gattungen nicht vorzukommen scheinen. Dazu gehört namentlich die mehrfach sich findende Abkürzung des vierten Dorsalstreifs, während der fünfte fast die Basis erreicht, und die eigenthümliche Gabelung des Suturalstreifs an der Spitze. H. connectens Lew., bei dem diese Gabelung sehr deutlich ist, ist in dieser Hinsicht nicht von subridens verschieden, denn auch von diesem sagt Marseul: "la suture est accompagnée au bout d'une courte striole." Es scheint ein der ganzen Gattung gemeinsames Merkmal zu sein, dass der Nahtstreif sich kurz vor der Spitze der Naht zuwendet; bei mehreren Arten ist er durch ein kurzes, die ursprüngliche Richtung beibehaltendes Stück ergänzt, welches sich zuweilen mit dem Nahtstreif verbindet und mit ihm eine Gabel bildet.

Die Stirn ist bei allen bekannten Arten nur neben den Augen gerandet. Das Halsschild besitzt einen, dem Rande sehr genäherten Lateralstreif, welcher jederseits hinter den Augen unterbrochen ist, der mittlere Theil oft hakenförmig zurückgebogen; der Marginalstreif ist sehr fein, tritt hinter den Vorderecken auf die Seitenkante, so dass er von oben nicht sichtbar ist, und erlischt bald. Möglich, dass er auch einigen Arten ganz fehlt (ridens? parensis? - die Angaben Marseul's sind unbestimmt). Dies eigenthümliche Ensemble der Streifen spricht sehr dafür, dass wir es hier mit einer recht natürlichen Gattung zu thun haben. Als typische Form der Gattung betrachte ich nicht die folgende in mancher Hinsicht abweichende Species, sondern subridens Mars. und die mit ihm verwandten Arten. 20. Hypobletus orbatus n. sp. - Rio Janeiro.

Parallelo-ovatus, subdepressus. rufo-brunneus. Fronte impressa, punctulata, lateribus striata. Thorace lateribus punctulis sparsis, stria marginali tenui, basi abbreviata, laterali margini propinqua in angulis anticis hamata, parte media pone caput a margine remota, late disjuncta, ante scutellum foveola impressioneque indistincta longitudinali. Elytris stria subhumerali dejecta, apicali, medium vix attingente, dorsalibus punctatis 3 integris $4^{\prime \prime}$ vix breviore $5^{\prime 2}$ medio suturali basi parum abbreviata. Propygidio sat fortiter denseque, pygidio basi punctato apice fere laevi. Prosterno sat lato, striis antice vix, basi breviter divergentibus, lobo marginato; mesosterno late sinuato, stria marginali integra; tibiis anticis 5- vel 6-denticulatis. - Long. $1^{4 / 5} \mathrm{~mm}$. 
Die Längenverhältnisse der Dorsalstreifen und vor allem der Subhumeralstreif unterscheiden diese Art von den verwandten. Der letztere liegt hart neben dem kräftigen, einzeln punktirten Epipleuralstreif und ist wegen seiner Feinheit leicht zu übersehen. Das Pygidium hat an der Basis eine ziemlich regelmässige, in der Mitte etwas zurücktretende Querreihe kräftiger augenförmiger Punkte, dahinter ist es unregelmässig, hinter der Mitte viel feiner, an der Spitze kaum punktirt. Die Art ist auch mehr gewölbt als die übrigen, mir bekaunten.

21.* Phelister Riouka Mars. Mon. 1861, p. 158.

22. Phelister dives Mars. Mon. 1861, p. 157. - Petropolis.

23. Phelister haemorrhous Mars. Mon. 1853, p. 476. Boa Sorta.

24. Phelister brevistrius Mars. Mon. 1853, p. 485. - S. Rita.

25. Phelister distractus n. sp. - Rio Janeiro.

Late ovatus, subconvexus, niger nitidus, pedibus brunneis; fronte subimpressa, minute punctulata, stria antice recta, utrinque ad oculum interrupta. Thorace lateribus sparse punctato, stria marginali pone caput interrupta, laterali tenui integra, foveola antescutellari. Elytris stria subhumerali externa vix dimidiata, dorsalibus $1-3$ integris $4^{x}$ suturalique subaequalibus ultra medium abbreviatis, $5^{\text {i }}$ dimidiata, juxta tertiam striam puncto basali, epipleuris sulcatis; propygidio fortiter dense, pygidio minus fortiter sparse punctatis. Prosterno striis convergentibus antice arcuatim basique junctis, mesosterno brevi, late sinuato, stria antica subinterrupta, postica arcuata margini approximata; tibiis anticis 5-denticulatis, - Long. $2 \frac{1}{2} \mathrm{~mm}$.

Die ganze Oberseite ist sehr fein und zerstreut punktulirt; die Punktirung des Halsschildes zieht sich von den Augen nach hinten, vom Seitenrande entfernt. Die Art steht dem farctus Mars. 1ahe, aber bei diesem ist der Grund des Pygidiums zwischen den gröberen Punkten sehr dicht punktulirt, während bei distractus nur einzelne feine Pünktchen eingestreut sind; ferner ist bei distractus der Lateralstreif des Halsschildes sehr viel feiner, dem Rande noch näher, der Stirnstreif jederseits unterbrochen u. s. w. Von gracilis Schm., mit welchem die Sculptur der Oberseite ebenfalls ziemlich übereinstimmt, unterscheidet sich die vorliegende Art, von der Grösse abgesehen, durch den Stirnstreif, andre Punktirung des Halsschildes und Pygidiums und die Streifen des Sternum.

Das Mesosternum ist von einer Ecke zur andern leicht ausgerandet, der Streifen tritt nach der Mitte zu ein klein wenig vom Rande zurück und deutet so den ganz allmählichen Uebergang zu den Arten mit zweibuchtigem Mesosternum an. 
26. Phelister suturalis n. sp. - Rio Janeiro.

Late ovatus, sat convexus, rufus nitidus, elytrorum dorso obscuriore. Fronte cum clypeo impressa, stria subtili integra antice subsinuata; thorace lateribus subtiliter punctulatis, punctis majoribus perpaucis, foveola antescutellari rotundata, stria marginali integra pone caput fortiore, laterali margini proxima in angulo desinente. Elytris stria subhumerali externa, dorsalibus 4 suturalique integris, quinta medium superante, epipleuris unistriatis. Propygidio sat fortiter nec vero dense, pygidio multo subtilius disperse punctatis, hoc apice laevi; prosterno angusto striis antice subparallelis arcuatim junctis basi divergentibus, basi recta; mesosterno vix sinuato stria marginali integra, postica suturae mesometasternali propinqua bisinuatim arcuata; tibiis anticis 6-7-denticulatis. -- Long. $2 \mathrm{~mm}$.

Auch hier ist die ganze Oberseite sehr fein, am feinsten auf den Flügeldecken punktulirt. Die etwas stärkeren, einzeln eingestreuten Punkte des Pygidiums sind auffallend schwächer als die des Propygidiums. Das Mesosternum ist ganz schwach ausgerandet, so dass es, etwas von hinten betrachtet, grade erscheint; der Lateralstreif des Halsschildes erreicht nicht den Augenwinkel, er ist an den Seiten dem Marginalstreif sehr genähert, der letztere hinter dem Kopfe stark und tief, so dass man ihn fast für den Lateralstreif halten könnte.

Eine ähnliche Deckensculptur besitzt nur rubens Mars., der aber keinen Lateralstreif und einen abgekürzten Subhumeralstreif besitzt. Berücksichtigt man den ganzen Subhumeralstreif, so gehört die Art neben Teapensis Mars., mit welchem sie nicht zu verwechseln ist.

Das einzige Exemplar ist möglicherweise nicht voll ausgefärbt.

27. Phelister daugar Mars. Mon. 1861, p. 167. - Rio Janeiro = Phelister Pauli Mars. Abeille 1864, p. 316.

Die Beschreibung dieser beiden Arten, die beide ganz richtig mit Celebius Mars. verglichen werden, ergiebt auch nicht den geringsten Unterschied. Die etwas andre Färbung des daugar ist sicher Folge mangelhafter Ausfärbung; auch meine Stücke, wie oft die Phelister variiren in dieser Hinsicht. In der Tabelle Abeille 1864 , p. 314 schreibt Marseul dem daugar allerdings 3 ganze Rückenstreifen zu, in der Beschreibung Mon. 1861, p. 167 heisst es aber ausdrücklich: "3e un peu interrompue au milieu." Auch der ganze dritte Streif würde übrigens keinen Unterschied begründen; das vorliegende Stück hat diesen Streif breit unterbrochen, ein andres brasilianisches Exemplar meiner Sammlung zeigt ihn fast ganz, bei einem dritten von Bahia ist er absolut vollständig. Bei der folgenden Art findet sich eine analoge Verschiedenheit des vierten 
Streifs, und Aehnliches kommt auch in andern Gattungen, wie z. B. Platysoma, vor.

28. Phelister carinifrons Schm., Ent. Nachr. 1893, p. 87. Rio Janeiro.

Ein anscheinend nicht normal gefärbtes Stück (Halsschild und Hinterwinkel der Flügeldecken rothbraun), welches die schon bei dor Beschreibung von mir ausgesprochene Vermuthung bestätigt, dass die Unterbrechung des vierten Streifs bei dem typischen Exemplar individuell sei. Dieser Streif ist beim vorliegenden Stück ganz, nur auf der linken Decke nahe der Basis ein wenig unterbrochen. Die rudimentären Prosternalstreifen finden sich hier dicht vor dem Prosternalfortsatz, während das typische Stück solche an der Basis besitzt.

Der Lateralstreif des Halsschildes ist bei dieser Art, namentlich nach der Basis zu, auffallend weit vom Rande entfernt.

29. Phelister sejunctus n. sp. - S. Rita.

Brevissime ovatus, lateribus parum rotundatus, subdepressus, niger, nitidus. ore pedibusque rufo-piceis. Fronte depressa punctulata, stria antice fortiore curvata, utrinque ad oculum interrupta. Thorace lateribus punctato ante scutellum obsolete foveolato, stria marginali antice interrupta, laterali forti, pone oculos breviter interrupta, parte media utrinque hamata. Elytris striis subhumeralibus duabus humerum attingentibus, externa sinuata, dorsalibus 3 integris, $4^{n}$ subabhreviata, $5^{a}$ medio, suturali ultra abbreviatis. Propygidio dense ocellatim. pygidio multo subtilius punctato et subtiliter mguloso. Prosterno basi sat lato, striis convergentibus antice arcuatim junctis, lobo marginato, subtiliter punctulato; mesosterno sinuato, stria marginali in medio intervupta, transversali arcuata margini antico proxima; tibiis anticis 5-dentatis. - Long. $2^{3 /} \mathrm{mm}$.

Diese Art ist neben subdepressus $\mathrm{Schm}$. zu stellen, mit welchem sie, trotz der ganz abweichenden Körperform, eine Reihe von Merkmalen gemein hat. Namentlich das Mesosternum, die Sculptur der Pygidien und auch die Subhumeralstreifen erinnern stark an die genannte Art. Der äussere Subhumeralstreif, der eigentlich nur bis zur Mitte reicht, ist durch ein kurzes mehr nach innen liegendes Stück ergänzt, welches auf der rechten Flügeldecke des einzigen Exemplars mit dem Apicaltheil verbunden ist und so einen geschwungenen Streifen bildet. Der Seitenrand des Halsschildes ist, namentlich vorn, etwas aufgewulstet, die Punktirung bildet ein breites, unregelmässiges, vom Rande entferntes Band. Die auf dem dichtrunzligen Grunde des mattglänzenden Pygidium's stehenden Punkte werden nach der Spitze zu feiner und weitläufiger. Der Lateralstreif 
des. Halsschildes ist wie bei Friburgicus Mars., welcher schon durch den einzigen Subhumeralstreif und obsoleten Stirnstreif sehr verschieden ist.

Die Dorsalstreifen, namentlich die inneren, sind bei sejunctus an der Basis ziemlich stark einwärts gebogen.

30. Phelister rufinotus Mars. Mon. 1861, p. 170. - S. Rita.

31. Phelister Fairmairei Mars. Mon.1861,p.172. - Rio Janeiro.

32. Phelister Salobrus Mars. Ann. Fr. 1887, Bull. p. CXLVIII. -- Petropolis, S. Rita.

33. Hister punctifer Pk. Mon., p. 25. - S. Rita, Petropolis.

34. Hister dubius Mars. Mon. 1854, p. 279. - Petropolis.

35. Epierus inscriptus n. sp. - Boa Sorta.

Rotunduto-ovatus, convexus, niger nitidus pedibus antennisque brunneis, clava rufa. Fronte impressa, subtiliter punctulata, clypeo in medio canaliculato. Thorace dorso subtiliter, lateribus densius fortiusque punctato, stria marginali integra, linea ante scutellum biarcuata. Elytris subtiliter punctulatis, stria subhumerali externa integra demissa, interna apicali dimidiata, dorsalibus 4 integris 5 et 6 basi abbreviatis, internis procipue crenatis, epipleuris tenuiter unistriatis. Propygidio parum fortiter, pygidio adhuc subtilius sat dense punctatis, hoc apice foveolato. Prosterno lato convexo, striis fere parallelis, mesosterno subrecto, stria antica breri in angulo, intermedia margini in medio proxima integra, postica punctata, valde anqulata; tibiis anticis dense brevissime spinulosis. - Long. $3 \mathrm{~mm}$.

Gehört zu der durch eine Scutellarlinie ausgezeichneten Gruppe des arciger, hastatus, coproüdes und epulo Mars. Die Linie vor dem Schildchen sowie das Sternum entspricht der von Marseul für coproüdes gegebenen Abbildung, nur sind die Prosternalstreifen noch mehr parallel und die hintere Querlinie des Mesosternum's mehr winklig; auch ist das letztere nicht zweibuchtig, sondern, von vorn gesehen, sogar leicht ausgerandet. Von allen beschriebenen Arten unterscheidet die vorliegende der innere Subhumeralstreif, der nur bei epulo ebenfalls vorhanden, dort aber ganz ist. Die inneren Dorsalstreifen erscheinen sehr deutlich kettenartig, indem ihr Rand jederseits mit einer Reihe eingestochener Punkte besetzt ist. . Die Punkte auch auf den Seiten des Halsschildes sind nicht dicht, aber tief eingestochen; etwa gleichstark, aber dichter die des Pygidium. Das Epistom nach der schmalen Mittelfurche hin abfallend.

36. Epierus angularis n. sp. - S. Rita.

Breviter ovatus, convexus, brunneo-piceus, nitidus; fronte convexa, minutissime punctulata; thorace lateribus fortius punctu- 
lato, stria marginali integra, antescutellari angulata. Elytris stria subhumerali externa demissa integra, interna basali, dorsalibus 4 integris $5^{2}$ et suturali basi abbreviatis, internis distinctius crenulatis, epipleuris unistriatis. Propygidio pygidioque sat dense sed subtiliter punctatis, hoc apice subfoveolato. Prosterno lato, striis utrinque divergentibus, mesosterno bisinuato, stria marginali interrupta, intermedia posticaque punctata parallelis curvatis; tibiis anticis dense breviter spinulosis. - Long. $2^{1} / 2 \mathrm{~mm}$.

Ebenfalls der Gruppe mit Scutellarlinie angehörig; dieselbe bildet hier einen nach vorn gerichteten stumpfen Winkel. Im Gegensatz zu sämmtlichen Arten der Gruppe ist bei angularis die Stirn gewölbt, wie bei den meisten Epierus. Die inneren Streifen sind ähnlich wie beim Vorigen gekerbt, aber nicht so breit. Von der Scutellarlinie abgesehen. steht die Art dem fissus Mars. recht nahe, besitzt namentlich auch den ziemlich $1 / 3$ erreichenden inneren Subhumeralstreif an der Basis; doch sollen bei fissus die beiden innersten Streifen fein, aber nicht abgekürzt sein, während sie bei angularis nur bis $\mathrm{zu}^{3 / 4}$ der Decken reichen.

37. Epierus mundus Er. Jahrb. 1834, p. 160. - S. Rita.

38. Epierus tersus Er. Jahrb. 1834, p. 161. - Rio Janeiro.

39.* Epierus Alberti Mars. Mon. 1861, p. 556.

40. Epierus bisbistriatus Mars. Mon. 1854, p.687. - S. Rita. 41.* Epierus fissus Mars. Mon. 1861, p, 559.

42. Epierus invidus Mars. Mon. 1861, p. 561. - Rio Janeiro. 43. Epierus Vandepolli Schm. Ent. Nachr. 1889, p. 371. Boa Sorta. 44. Epierus Mariae Mars. Mon. 1861, p. 564. Rio Janeiro.

Obwohl das Fpistom nicht zweizähnig, sondern einfach ist, glaube ich mich in der Bestimmung nicht zu irren. Das eigenthümlich zerstreut punktirte Halsschild und die übrigen Merkmale lassen kaum einen Zweifel zu und es stimmt nur mit dem, was wir sonst bei den Epierus sehen, dass nur das $\sigma^{7}$ ein gezähntes Epistom besitzt. Bei dem $q$ ist also die Stirn flach, vertieft, das Epistom viel mehr concav und diese Vertiefung nach der Stirn zu durchaus gradlinig begrenzt, so dass es fast scheint, als sei Stirn und Epistom durch einen Streifen getrennt, ähnlich wie beim ostindischen Beccarii Mars. 45. Epierus lucidulus Er. Jahrb. 1834, p. 161. - S. Rita, Petropolis, Boa Sorta.

46. Epierus notius Mars. Mon. 1861, p. 560. - S. Rita.

47. Hetaeriosoma Sahlbergi Schm. Deutsch. Ent. Zeit. 1893, p. 185. - Petropolis.

Ich habe dieses Thier, wie ich schon a. a. 0 . bemerkte, vorweg- 
genommen, um es mit einem Dutzend andrer myrmecophiler Histeriden aus Südamerika zusammen beschreiben zu können. Es ist unstreitig die interessanteste Art der ganzen Collection, unseren Hetaerius, mit welchen sie schon der Fühlerkeule wegen nicht vereinigt werden kann, sehr ähnlich.

48. Carcinops misella Mars. Mon. 1855, p. 95. - Petropolis.

4.9. Carcinops troglodytes Payk. Mon., p. 46. - S. Rita.

50. Paromalus causticus Mars. Mon. 1862, p. 20. - Rio Janeiro.

51. Paromalus concentricus Mars. Ann. Soc. Ent. Belg. 1870,

p. 100. - Petropolis.

52. Paromalus trifolium Mars. Mon. 1861, p. 21.?-Boa Sorta.

Obwohl das Stück mit einem angeblich von Marseul bestimmten Exemplar der alten Hag'schen Sammlung übereinstimmt, bin ich nicht sicher, ob es in der That dieser Art angehört.

53. Paromalus irregularis n. sp. - Petropolis.

Ovalis, subconvexus, niger nitidus; fronte subdepressa, punctulata, stria integra, antice curvata clypeum ambiente. Thorace minute parum dense punctato, stria marginali integra; elytris adhuc sparsius vix fortius punctatis, sutura antice subelevata, striola obliqua basali sat distincta, suturali tenui medium paulum superante, apice quoque subabbreviuta. Propygidio basi media obsolete biimpressa, dimidio antico (margine angusto basali laevi excepto) dense sat fortiter punctato. postico punctulis raris vix perspicuis; pygidio dense punctulato 우 sulco profundo bilobo, saepius plus minusve imperfecto. Prosterno cum lobo vix perspicue sparse punctulato, mesosterno stria biangulata, parte media paulum latiore, angulis impressis, paululum productis; metasterno lateribus distincte, nec v'ero dense punctato. Tibiis anticis quadridenticulatis. - Long. $1^{3 / 4}-2^{1 / 4} \mathrm{~mm}$.

In die Nähe des trifolium Mars. zu stellen, aber nicht flach und parallel, sondern oval, leicht gewölbt, etwa wie ein recht kleiner cordipygus Mars. An der Punktirung des Propygidium leicht kenntlich; dieselbe bildet eine Art Band, welches vorn einen schmalen Rand, hinten etwa dic hintere Hälfte freilässt, und auch die Seiten nicht erreicht. Die Geschlechtsauszeichnung des of ist sehr variabel. Sie besteht aus 2 schräg nach innen liegenden unregelmässigen Ovalen, die vorn nur durch einen schmalen Kiel getrennt sind. So vollständig ¡st die Figur aber selten, es verschwinden hie und da Theile, so dass manchmal nur ein paar unregelmässige divergente Linien übrig bleiben, oder die Spitzen vereinigen sich zu einer dem Apicalrand des Pygidium's folgenden Furche und es entsteht eine Art umgekehrt herzförmiger Figur, die fast das ganze Pygidium einnimmt. $P$. oculi- 
pygus Mrs. scheint eine ähnliche Sculptur des Pygidium's zu besitzen, ist aber sonst sehr verschieden.

54. Paromalus addendus n. sp. - Petropolis.

Praecedenti simillimus, sed minor, oblongo-ovatus, elytris fortius punctatis, stria suturali paulolongiore. - Long. $1^{2 / 3}-1^{3 /}{ }_{4} \mathrm{~mm}$.

Dem Vorigen so nahestehend, dass ich Bedenken trug, ihn zu trennen, zumal auch die Geschlechtsauszeichnung sehr ähnlich ist Er ist aber kleiner und vor Allem von viel schmalerer Körperform; die Punktirung der Oberseite ist etwas kräftiger, namentlich auf den Flügeldecken, und der Nahtstreif ist ein wenig länger. Die Furchen des Pygidium's bilden eine ähnliche Figur wie bei dem Vorigen und ändern ebenfalls sehr ab.

55. Saprinus azureus Sahlb. Peric. Ent.p. 4. - S. Rita, RioJaneiro. 56. Saprinus modestus Er. Jahrb. 1834, p. 187. - Petropolis, S. Rita.

57. Saprinus modestior Mars. Mon. 1855, p. 493. - Rio Janeiro. 58. Saprinus innubus Er. Jahrb. 1834, p. 187. - S. Rita.

5.9. Saprinus arcipygus Schm. Ent. Nachr.1890,p.44. - S. Rita. 60.* Saprinus dolatus Mars. Mon. 1862, p. 482.

61. Teretriosoma cyaneum Lew. Ann. a Mag. N. H. 1889, p. 287. - Boa Sorta.

Bei dem einen Geschlecht, wahrscheinlich dem $\sigma$, ist der Fühlerschaft mit langen, gelben Haaren besetzt, die Stirn vorn mit einem rundlichen Eindruck, bei dem andern an derselben Stelle mit einer flachen, etwas dreieckigen Erhabenheit.

62. Bacanius scalptus Lew. Biolog. Central. Am. Col. II, 1, p. 237. - S. Rita.

63. Bacanius convergens n. sp. - Petropolis.

Rotundato-ovatus convexus, brunneus, nitidus. Fronte plana sparse minutissime, thorace minute sparsim punctulato, angulis anticis acutis, stria marginali integra. Elytris apice tantum distinctius punctulatis, stria subhumerali demissa integra, dorsali prima basi abbreviata, epipleuris striola abbreviata. Propygidio pygidioque sparse minute punctulatis. Prosterno subquadrato, lobo magno minute ruguloso punctulatoque, mesosterno antice sublisinuato lateribus marginato metasternoque laevi; tibiis anticis curvato-dilatatis unidentatis, posticis minus subangulatim dilatatis. - Long. vix $1 \mathrm{~mm}$.

Die Angaben über die Punktirung beziehen sich auf mikroscopische Vergrösserung, unter der Lupe erscheint das Thier glatt. Auch bei starker Vergrösserung sind die Punkte äusserst fein und einzeln auf der Stirn und dem vorderen Theil der Flügeldecken, besser sichtbar 
66 Joh. Schmidt: von Herrn Prof. Sahlberg ges. Histeriden.

an der Spitze der letzteren und auf dem Halsschild, namentlich dem Grunde zu, etwas feiner auf dem Pygidium und Propygidium. Der Prosternalfortsatz ist mit einzelnen Pünktchen auf maschenartig gestricheltem Grunde besetzt; diese Sculptur setzt sich auch auf den vorderen Theil des Prosternum's selbst fort, dessen Streifen kaum merklich convergiren. Der innere Streif der Flügeldecken hört in $3_{4}{ }_{4}$ der Länge auf, der äussere, der etwas unterhalb des Seitenrandes liegt, ist ganz, beide nach aussen etwas scharfkantig.

Die Art steht dem humicola Mars. sicherlich nahe, dieser soll aber keinen Dorsalstreif auf den Flügeldecken besitzen; noch ähnlicher scheint er dem nordamerikanischen debilitans Casey zu sein, doch ist dieser viel kleiner und das Sternum anders punktirt. 64. Acritus Floridae Mars. Mon. 1862, p. 693. - Petropolis.

Ein Exemplar, welches mit nordamerikanischen Stücken aus Carolina und Georgia aufs Genaueste übereinstimmt. 


\section{$2 \mathrm{BHL}$ Biodiversity Heritage Library}

Schmidt, Joh. 1896. "Aufzählung der von Herrn Professor F. Sahlberg in Brasilien gesammelten Histeriden." Berliner entomologische Zeitschrift I herausgegeben von dem Entomologischen Verein in Berlin 41, 55-66.

View This Item Online: https://www.biodiversitylibrary.org/item/34409

Permalink: https://www.biodiversitylibrary.org/partpdf/82760

\section{Holding Institution}

Smithsonian Libraries

\section{Sponsored by}

Smithsonian

\section{Copyright \& Reuse}

Copyright Status: NOT_IN_COPYRIGHT

This document was created from content at the Biodiversity Heritage Library, the world's largest open access digital library for biodiversity literature and archives. Visit BHL at https://www.biodiversitylibrary.org. 